\title{
Modifying children's processing of categorizable information for memory
}

\author{
JAMES W. HALL and SHARON C. MADSEN \\ Northwestern University, Evanston, Illinois 60201
}

\begin{abstract}
Twenty children in each of Grades 3 and 5 were given training over nine sessions in the use of organizational strategies for encoding and retrieval. A second group at each age level was given sheer practice on the materials used in the strategy training, and a third group was untreated. Organizational strategy training resulted in higher levels of recall, clustering, and study organization on posttests in which the children studied an array of categorizable pictures and an array of categorizable words. This was true both for test items from categories and items not used and for those that were used in training. Such training produced greater clustering but not greater recall for an aurally presented list of categorizable words.
\end{abstract}

This experiment is concerned with the modifiability of children's processing of categorizable information. There are a variety of mechanisms that might account for the usual superiority of recall for categorizable vs. unrelated material, varying in their locus (encoding vs. retrieval) and automaticity (automatic vs. strategic). Postman (1972) has described several of these mechanisms and suggested that they each are likely to play some role in situations involving the recall of categorizable items. In the case of children, however, the available data indicate that elementary school age children are relatively deficient in the spontaneous initiation of strategies both for encoding (Neimark, Slotnick, \& Ulrich, 1971) and retrieving (Kobasigawa, 1974) categorizable information. Whether such deficiencies are maturationally determined or due to insufficient relevant experiences is a question of interest both from a theoretical and a more immediate practical (educational) view and is the focus of the present study.

It seems clear that children at least as young as about 7 years will implement organizational strategies for retrieval (Scribner \& Cole, 1972) or for storage (Moely, Olson, Halwes, \& Flavell, 1969) of category information when explicitly instructed to do so, but do not do so spontaneously. Can such strategies be acquired by children through training? The evidence to date has been unconvincing. After having imposed an exhaustive category-search strategy on children in Grades 2, 4, and 6 for four successive study-recall trials with a categorizable word list, Scribner and Cole (1972) found no generalization to a new word list. Moely and Jeffrey

We are grateful to our research assistants, Cheryl Gay, Jerry Goldman, Lisa Grossman, Gay Hall, Sue Hendricson, and Nancy Naron, and to the school officials, teachers, and students of the Martin Luther King Elementary School in Evanston. The research was supported by Grant OEG-5-7-0051 from the U.S. Office of Education. Requests for reprints should be addressed to James W. Hall, School of Education, Northwestern University, Evanston, Illinois 60201.
(1974) reported an experiment in which one group of 6 and 7 year olds were given instruction and practice in sorting pictures into categories and producing category labels during study. They then were told to think of the category labels and search them during the test stage. A control group was given practice without such instruction. Next, a second picture recall task was given using new material, presumably to examine the generalized effects (transfer) of the earlier training. At that time the trained subjects were told to "try and find things that are alike or go together and remember them together," whereas the controls were told to "try real hard to remember a lot of things." Recall on the transfer task was superior for the trained group and the authors concluded that they had demonstrated an effect of teaching organizational techniques that generalized to new items, improving recall on those items. However, that conclusion is unjustified, since training differences were confounded with differences in transfer test instructions. In fact, a subsequent experiment (Hall, 1976) in which only the transfer test instructions were varied produced results essentially identical to those of the Moely and Jeffreys study.

Hall and Madsen (Note 1, Experiment 3) provided 26 training sessions in which preschoolers were given instruction and practice in searching for conceptual relationships, producing category labr's, and employing category labels as retrieval cues. Despite the intensiveness of that training, the children = - rformed no better on subsequent memory tests with categorizable material not used in training than did a control group that received no training. These negative results led us to the present experiment, in which the effects of organizational encoding and retrieval strategy training were examined in children in Grades 3 and 5.

\section{METHOD}

Subjects, Design, and Materials

In each of Grades 3 and 5, 60 children were assigned to 
three groups using random procedures except to insure 10 boys and 10 girls per group. Two groups participated in nine training sessions, with the third group serving as a passive control (PC) group (i.e., receiving no training whatsoever). For the experimental training (ET) condition, a set of exercises, based on standard laboratory learning and memory tasks, was developed that emphasized attention to conceptual relationships, production of category labels, organization by category during study, and the use of category information to assist in recall and recognition. The content consisted of common instances of 16 categories preselected from a larger pool of 25 categories judged to be familiar to most school children. The remaining nine categories were withheld until the test phase, then used to examine the generalization of training effects to "new" categories. The categories used in training (old categories) were: appliances, birds, countries, dwellings, earth formations, flowers, fruits, furniture, insects, land animals, measures of time, parts of buildings, sea animals, sports equipment, tools, and vegetables. The new categories were: beverages, body parts, clothing, colors, money, occupations, relatives, vehicles, and weather phenomena.

The remaining condition, the control training (CT) condition, was included to evaluate the effects of simple practice on the training tasks. The CT subjects were given approximately equal familiarity with the items used during training to the ET condition, but without any instructions concerning the conceptual relations among items.

Following the training period, training effects were examined in a series of four individual test sessions beginning approximately 12-20 days after each trained subject's final training session, and occurring at intervals of about 12-16 days. The test tasks were administered by an experimenter who had not worked with the child during training and was aware of the subject's grade level but not aware of the condition to which he was assigned. Test procedures were identical for all 120 subjects and the testers made no reference during testing to previous training.

\section{Training Tasks and Procedures}

Training occurred in seven individual and two group sessions distributed over a 10-week period. The sessions ranged from about 15 to $35 \mathrm{~min}$ in length for the ET group and 5-10 min less for the CT condition and were conducted by five experimenters, each responsible for four ET and four CT children per grade level. The content and procedures were controlled by scripts prepared by the authors, insuring high, although not perfect, uniformity across experimenters within conditions.

Altogether, seven different types of training tasks were employed with the ET and CT subjects: (1) study and recall of a random array of categorizable pictures, (2) recall of aurally presented unblocked categorizable word lists, (3) recognition of categorizable words, (4) a word association test modified for ET subjects such that category labels were requested as responses, (5) recall of words from prose passages containing several instances of each of several categories, (6) recall of words from two consecutively presented categorizable lists and differentiation between the lists, and (7) a paired associate task in which each of three response terms was paired with three different members of the same category (e.g., TONY was paired with HOUSEBOAT, HUT, and CABIN). The CT subjects were simply given practice on the various tasks, whereas the ET subjects also were repeatedly given instruction regarding attention to and use of category information to assist their performance. In addition to the above tasks, in Session 1 the ET subjects received an explanation of the concept of a category, and in Session 8 both groups were asked to explain how they went about learning and remembering a set of categorizable words. Finally, in Session 9 each ET and CT child was asked to administer a picture-recall task to the experimenter.

\section{Test Tasks and Procedures}

Two test tasks were administered in each of the first three test sessions. In each of these sessions, the first test was a word recall task in which 18 words were presented by tape recorder at the rate of 1 item every $4 \mathrm{sec}$. Then, following a 20 -sec filler task, a 90 -sec period was given for recall. Two such trials were given, with the order of items varying randomly across trials. For each word recall task, the list consisted of three words from each of two categories and four words from each of two other categories, plus four unrelated filler words. The only difference among these three tests was in the items used. In the first test (Session 1), the words and categories were ones not used during training (i.e., were New-New). The second test employed new items from old (training) categories (New-Old). In the third test the items were Old-Old.

The second task in each of the first three test sessions was a picture study-recall task similar to that used by Neimark et al. (1971). Twenty pictures of common objects were arranged before the child in a random order consisting of five rows and four columns. The pictures were of common objects from four categories, with four members of two of the categories and five members of the remaining two categories. Two unrelated filler pictures also were included. In the first test session, the items were New-New, in the second they were New-Old, and in the third they were Old-Old.

For each test the child was told to study the picture array and that he could rearrange the pictures however he wished to help his memory. After 3 min the pictures were removed and the child was given a 20 -sec nonverbal filler task followed by recall.

The final test session consisted of a single word study-recall task in which 20 words were presented, each on a $2 \times 6$ in. card. The procedure was identical to that for the picture studyrecall tests except that: (1) each child read each word aloud prior to receiving study instructions, and (2) an additional $30 \mathrm{sec}$ of study time was given. Nine of the words were New-Old and nine were New-New, plus two unrelated filler words.

\section{RESULTS}

Separate analyses of variance were applied to total recall scores and clustering scores for each of the seven tests and to the study organization scores for each of the four study-recall tests. These analyses examined the effects of grade level (G), conditions (C), and sex (S). For the word recall tests, changes from Trial 1 to Trial 2 (T) also were examined. Consistent with the major purpose of the experiment, in considering results our focus will be on main effects of the conditions variable and on interactions involving that variable. Also, since the groups were compared on a number of different measures, our chief interest was in the consistency with which particular significant effects were found. Table 1 shows mean recall clustering, and study organization scores. The two grade levels are combined in that table, since no pattern of interactions involving conditions and grade level was found.

\section{Recall}

The main effect of conditions did not approach significance in any of the three word recall tests. The Grade by Conditions interaction for recall of Old-Old 
Table 1

Mean Recall, Clustering, and Study Organization (SO)

\begin{tabular}{|c|c|c|c|c|c|c|c|c|c|c|}
\hline \multirow{2}{*}{$\begin{array}{c}\text { Test } \\
\text { Task }\end{array}$} & \multirow[b]{2}{*}{ Measure } & \multicolumn{3}{|c|}{ Experimental Training } & \multicolumn{3}{|c|}{ Control Training } & \multicolumn{3}{|c|}{ Passive Control } \\
\hline & & New-New & New-Old & Old-Old & New-New & New-Old & Old-Old & New-New & New-Old & Old-Old \\
\hline Word & Recall & 9.29 & 8.25 & 9.48 & 9.40 & 8.40 & 9.31 & 9.61 & 8.32 & 9.05 \\
\hline Recall & Clustering & 1.20 & 1.23 & 1.35 & .88 & .67 & .74 & .94 & .23 & .32 \\
\hline Picture & Recall & 14.02 & 12.88 & 15.48 & 11.85 & 11.28 & 13.32 & 12.80 & 10.70 & 12.45 \\
\hline Study & Clustering & 2.47 & 2.26 & 2.74 & 1.18 & 1.16 & 1.57 & .80 & .66 & 1.23 \\
\hline Recall & SO & 4.42 & 4.22 & 5.00 & 2.52 & 2.85 & 2.98 & 2.70 & 2.45 & 2.90 \\
\hline Word & Recall & & 14.70 & & & 13.95 & & & 12.68 & \\
\hline Study & Clustering & & 2.34 & & & 1.36 & & & 1.41 & \\
\hline Recall & SO & & 4.15 & & & 2.72 & & & 2.42 & \\
\hline
\end{tabular}

items was marginally reliable $[\mathrm{F}(2,108)=3.10, \mathrm{p}=.05]$, reflecting the fact that at Grade 3 the recall was highest for the CT group and lowest for the PC group, whereas the reverse was true at Grade 5, variations that we suspect were due to sampling error. No other interactions involving conditions were significant.

In contrast, recall was affected by training in each of the picture study-recall tests and in the word studyrecall test $[F(2,108)>3.20, p<.05$, in each case $]$. For each of these four tests and at both grade levels, the ET condition displayed higher recall than each of the remaining conditions. As may be seen in Table 1, for two of the four tests (collapsing over grades) the TC group was lowest in recall, whereas for the remaining two the PC group was lowest. For each test, recall increased with grade $[F(1,108)>20, p<.01$, for each]. For the three picture tests, but not for the word test, the recall differences among conditions was significantly greater for girls than for boys $[\mathrm{F}(2,108)>3.60$, $\mathrm{p}<.05$, for each]. No other effects were significant. Clearly, the experimental testing facilitated recall on the study-recall tasks and the facilitation extended to items from categories never employed during training, and apparently was somewhat greater for the females. Recall was not affected for the word recall tasks, and there is no evidence that the simple practice given the CT subjects had any effect on their recall on any of the seven test tasks.

\section{Clustering}

Both the runs test (Frankel \& Cole, 1971) and the item clustering index (Robinson, 1966) were applied to the study-recall test data with highly similar results; the correlations between the two measures approximated .90 . The results using only the runs test, in which degree of clustering is expressed in terms of $\mathrm{z}$ scores, are reported here.

As shown in Table 1, the clustering data parallel the recall data with one important exception. That exception occurs in the word recall tests, where clustering was greater for the ET than for the remaining conditions. For the New-Old and the Old-Old tests (but not the New-New test), the main effect of condition was significant $[F(2,108)=5.90, p<.01$, for each $]$.
For each of the four study-recall tests, the main effect of conditions was significant $[F(2,108)>7$, $\mathrm{p}<.01$, for each]. In each case clustering was greater for the ET group. For the New-New and Old-Old picture study-recall tests, the Conditions by Sex interactions were significant $[\mathrm{F}(2,108)>3.50, \mathrm{p}<.05$, for each $]$. Girls displayed more clustering than boys in the ET condition and less in the CT condition. No other effects involving conditions were significant.

\section{Study Organization}

Organization during study was quantified on a 6-point scale by a judge blind to the condition of each subject. A score of 1 was assigned for no rearranging and a score of 6 for perfect category rearrangement. Scores between these values reflected varying degrees of categorical organization.

Organization of items by category during study was substantially and consistently greater for the ET children, with little difference between the CT and PC groups. This was reflected in significant main effects of conditions for each of the four study-recall tests $[F(2,108)>10, p<.01$, for each test $]$. The ET children had acquired a study strategy that they put to use with new material, and that strategy did not evolve by the sheer practice of the CT children.

\section{DISCUSSION}

Several conclusions are indicated by these results. First, for children in this age range (9-13 years), instruction such as that provided the ET group can produce sL sstantial effects on memory performance involving conceptualy related material, in contrast to our previous experience with $\mathrm{F}^{-}$.schoolers. The power of these effects is evidenced by the $r$ generality (to material not used in training) and ersistance (over at least a period of several weeks) in the case of the study-recall tasks. Accompanying the increase in recall accuracy was increased categorical organization during study and clustering during recall, adding weight to the conclusion that effective encoding and retrieval strategies had been acquired by the ET children. Second, sheer practice in encoding and retrieving categorizable information does not result in powerful effects. In fact, there was scant evidence of any effect of such practice.

Third, the extent of training effects is highly dependent on particular task characteristics. Such effects were powerful when the task allowed the children to fully capitalize on the information they had acquired during training and when the 
acquired strategy involved physical manipulation of the to-beremembered material. Such was the case in the picture and word study-recall tasks, but not in the word recall task. In the latter task, item presentation was controlled by the experimenter, relatively heavy demands on short-term memory were made for those who attempted to organize material by category during encoding, and no opportunity for a physical representation of the categories was provided.

Although recall on the word recall tests was not increased by training, clustering during recall was increased somewhat. A possibility suggested by that finding (although not the only one) is that the trained subjects were more likely to search categories during recall, but because the presentation conditions did not allow sufficient opportunity to extensively organize by category during encoding, the category search proved no more effective for retrieval than processes employed by the control subjects. The failure for the effect on clustering to be accompanied by a corresponding increase in recall again underscores the danger in assuming a causal relationship between clustering and recall.

\section{REFERENCE NOTE}

Hall, J. W., \& Madsen, S. C. Children's learning of categorizable information. Paper presented at the annual meeting of the American Educational Research Association, Chicago, April 1974.

\section{REFERENCES}

Frankel, F., \& Cole, M. Measures of category clustering in free recall. Psychological Bulletin, 1971, 76, 39-44.

HALL, J. W. A reexamination of the effect of organization training on children's recall of categorized items. Child Development, 1976, 47, 1211-1213.

Kobasigawa, A. Utilization of retrieval cues by children in recall. Child Development, 1974, 45, 127-134.

MoEly, B. E., \& JEFFREY, W. E. The effect of organization training on children's free recall of category items. Child Development, 1974, 45, 135-143.

Moely, B. E., Olson, F. A., Halwes, T. G., \& Flavell, J. H. Production deficiency in young children's clustered recall. Developmental Psychology, 1969, 1, 16-34.

Neimark, E., Slotnick, N. S., \& Ulrich, T. Development of memorization strategies. Developmental Psychology, 1971, 5, 427-432.

Postman, L. A pragmatic view of organization theory. In E. Tulving \& W. Donaldson (Eds.), Organization of memory. New York: Academic Press, 1972.

Robinson, J. A. Category clustering in free recall. Journal of Psychology, 1966, 62, 279-285.

SCRIBNER, S., \& Cole, M. Effects of constrained recall training on children's performance in a verbal memory task. Child Development, 1972, 43, 845-857.

(Received for publication January 20, 1978.) 